\title{
Kardiale Ursachen von Hirn- infarkten: Vorhofflimmern und offenes Foramen ovale
}

Lorenz Reill und Harald Darius

Vivantes Klinikum Neukölln, Klinik für Kardiologie und konservative Intensivmedizin, Berlin

psychoneuro 2005; 31 (5): 270-273

Vorhofflimmern (VHF) und offenes Foramen ovale sind die häufigsten Ursachen für kardioembolische Schlaganfälle. Klinische Prädiktoren eines hohen systemischen Thrombembolierisikos und damit auch Schlaganfallrisikos sind zunehmendes Alter, vorheriger Schlaganfall, Diabetes mellitus, arterielle Hypertonie, vorheriger Myokardinfarkt und Vorliegen einer Herzinsuffizienz. Zur Prophylaxe hat sich eine dauerhafte dosiaadaptierte Antikoagulation bewährt, die alleinige Gabe von Azetylsalizylsäure hat sich als nur gering effektiv erwiesen. Die Kombination von Clopidogrel und Azetylsalizylsäure sowie neue Medikamente, z.B. Faktor-XaAntagonisten, werden zurzeit noch untersucht. Bei Patienten mit Schlaganfall und Foramen ovale oder bei Vorliegen einer Kontraindikation zur Antikoagulation besteht nach einer Embolie heute die Indikation zum Verschluss des Foramen.

I m Gegensatz zum Myokardinfarkt ist die Pathogenese des Schlaganfalls sehr heterogen, und ein bedeutender Anteil der ischämischen Schlaganfälle hat eine kardiale Ursache. Dabei handelt es sich um die kardioembolischen Schlaganfälle, deren Ursache entweder in morphologischen Strukturveränderungen des Herzens begründet ist (Tab. 1) oder auf Rhythmusstörungen beruhen. Aufgrund der Vielzahl der möglichen Ursachen sollen hier lediglich das Vorhofflimmern als häufigste Herzrhythmusstörung und rhythmogene Ursache kardiogener Thrombembolien sowie das offene Foramen ovale angesprochen werden.

\section{Vorhofflimmern:}

Ursache und Wirkung

Die häufigste Ursache des Vorhofflimmerns (VHF) ist die arterielle Hypertonie mit linksventrikulärer Hypertrophie und verzögertem Einfluss des Blutes über die Mitralklappe und dadurch bedingter atrialer Dilatation. Andere häufige Ursachen sind u.a. die Mitralklappenin- suffizienz und die Hyperthyreose. In Deutschland leiden etwa $0,5 \%$ der erwachsenen Bevölkerung an VHF mit deutlicher altersabhängiger Steigerung der Prävalenz auf bis zu $10 \%$ bei den über 80 -Jährigen. Die hämodynamischen Folgen des VHF bestehen in dem Verlust der Vorhofkontraktion, die zu ca. 20\% zum Herzzeitvolumen beiträgt, einer verschlechterten Belastungsadaptation und der Neigung zu tachykarden oder bradykarden Phasen, die zu eingeschränkter Belastbarkeit, Auslösung kardialer Ischämien und Synkopen führen können.

Neben den Problemen mit Herzrhythmus und -frequenz besteht die Gefahr der Thrombenbildung im mechanisch inaktiven linken Vorhof während anhaltender paroxysmaler (Dauer $<7$ Tage) oder persistierender Vorhofflimmerphasen (Dauer $>7$ Tage; nicht selbst limitierend).

Klinische Prädiktoren eines hohen systemischen Thrombembolierisikos und damit auch Schlaganfallrisikos sind zunehmendes Alter, vorheriger Schlaganfall, Diabetes melli- tus, arterielle Hypertonie, vorheriger Myokardinfarkt und Vorliegen einer Herzinsuffizienz.

Bei Patienten mit paroxysmalem oder persistierendem VHF, welches noch nicht in permanentes VHF übergegangen ist, kann eine medikamentöse oder elektrische Kardioversion versucht werden. Damit versucht man die hämodynamische Koordination zwischen Vorhof und Ventrikel wiederherzustellen, um die Patienten vor Tachy-Bradykardieepisoden zu schützen und eine bessere Belastungsadaptation zu gewährleisten. Nachdem man früher davon ausging, dass diese Patienten nach einer Vorbehandlungsphase von vier Wochen mittels Antikoagulation, einer erfolgreichen Kardioversion und einer Antikoagulationsbehandlung für vier bis sechs Wochen nach der Kardioversion ausreichend vor thrombembolischen Ereignissen geschützt sind, konnte mittlerweile durch Studien belegt werden, dass auch bei diesen Patienten eine dauerhafte Antikoagulation erforderlich ist.

Medikamentöse Schlaganfallprophylaxe

Die Effekte einer Antikoagulationsbehandlung im Vergleich zur Plazebobehandlung oder Acetylsalicylsäure auf die Schlaganfallund systemische Thrombembolierate sind in zahlreichen klinischen Studien bei Patienten mit sogenanntem „non-valvular atrial fibrillation“ untersucht worden. Dabei handelt es sich um Patienten, bei denen 
Abb. 1 Wirksamkeit einer Dosis-adaptierten oralen Antikoagulation

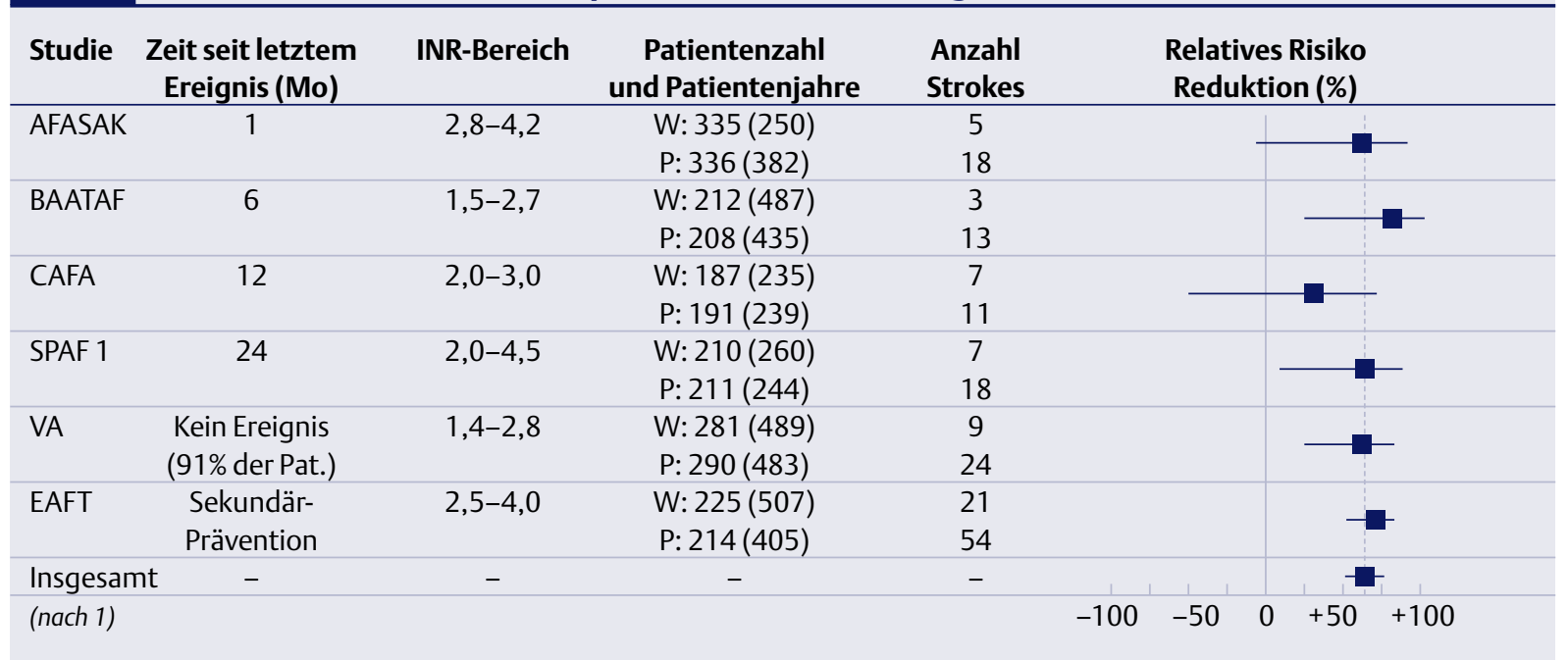

Metaanalyse kontrollierter randomisierter und Plazebo-kontrollierter Studien zur Wirksamkeit einer Dosis-adaptierten oralen Antikoagulation bei Patienten mit nicht-valvulären Vorhofflimmern

echokardiografisch eine klinisch signifikante Herzklappenerkrankung rheumatischer oder degenerativer Genese ausgeschlossen worden war. Die Metaanalysen der verschiedenen Studien zeigen dabei eine ca. 67\%ige Risikoreduktion für das Auftreten von Schlaganfällen und systemischen Thrombembolien bei einer Therapie mit Vitamin-K-Antagonisten (fast immer wurde Warfarin untersucht) mit einer INR zwischen 23 (Abb. 1). Untersuchungen mit höheren INR-Werten haben deutlich erhöhte Blutungskomplikationsraten, insbesondere zerebrale Einblutungen gezeigt. Sehr niedrig dosierte Antikoagulation (2 mg Warfarin/Tag, INR 1,0-1,2) zusammen mit ASS hat eine deutlich schlechtere klinische Effektivität als die dosisadaptierte Antikoagulation mit einer INR von 2-3 ergeben.

Aufgrund der geringen therapeutischen Breite der Vitamin-K-Antagonisten sowie der schwierigen Pharmakodynamik mit verzögertem Wirkungseintritt und Wirkungsende werden seit vielen Jahren alternative Therapiemöglichkeiten untersucht. Die alleinige Gabe von Acetylsalicylsäure hat sich dabei als nur gering effektiv erwiesen, in Metaanalysen konnte für Dosierungen $\geq 300 \mathrm{mg} / \mathrm{d}$ eine Reduktion der Rate an Schlaganfällen und systemischen Thrombembolien um ca. 20\% nachgewiesen werden, d.h. nur jedes fünfte Ereignis konnte effektiv verhindert werden, und bei vier von fünf Patienten trat der Schlaganfall trotz Prophylaxe auf. Im Rahmen einer großen wissenschaftlichen Studie (ACTIVE-Studie) wird derzeit bei 14000 Patienten mit permanentem oder paroxysmalem Vorhofflimmern untersucht, ob die kombinierte Gabe zweier Thrombozyteninhibitoren (Acetylsalicylsäure und Clopidogrel) ähnlich effektiv wie die dosisadaptierte Antikoagulation ist. Ebenfalls im Rahmen wissen- schaftlicher Studien werden derzeit neue Antikoagulantien nämlich direkte Thrombinantagonisten und Faktor-Xa-Antagonisten untersucht, die aufgrund ihres Wirkungsmechanismus eine effektive Inhibition der Thrombusbildung bei geringerer $\mathrm{Ne}-$ benwirkungsrate und akzeptabler therapeutischer Breite erwarten lassen. Für die am weitesten entwickelte Substanz mit innovativen Wirkungsmechanismus, Ximelagatran, konnte bereits im Rahmen zweier

\section{Abb. 2 Framingham-Risk-Score mit Angabe des Schlaganfall- risikos bei Patienten mit Vorhofflimmern}

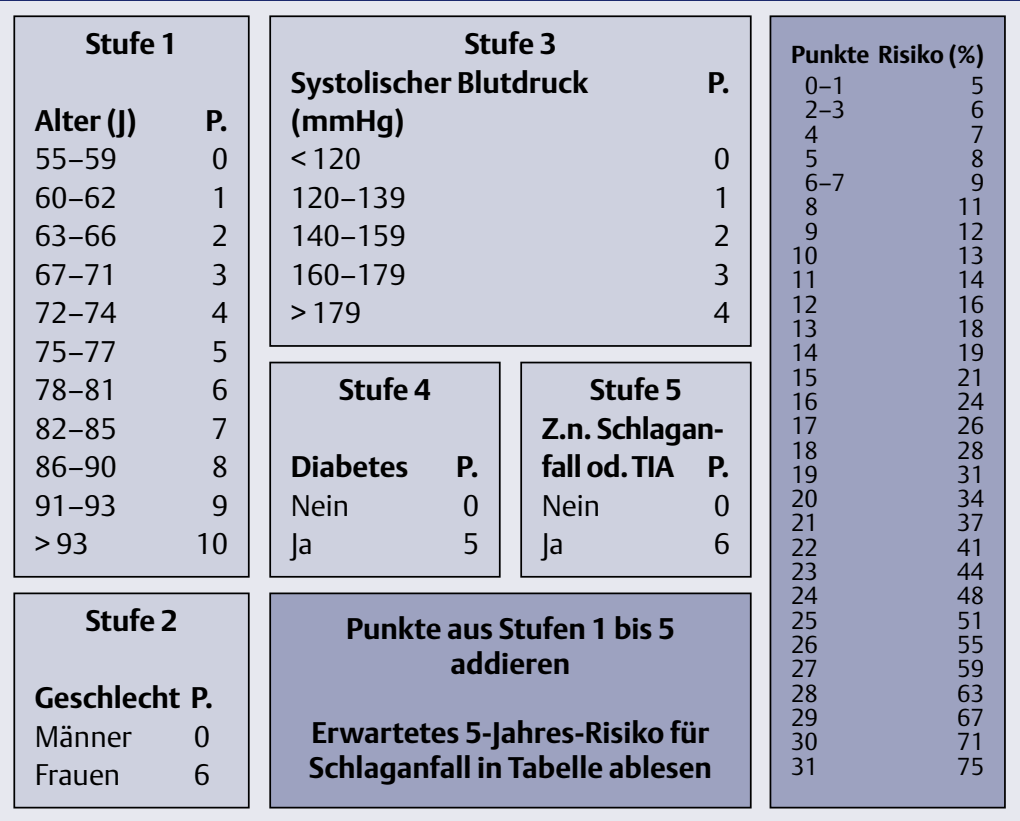


Tab. 1 Morphologische Ursachen kardioembolischer Schlaganfälle

- Akut oder chronisch veränderte Aorten- oder Mitralklappen nach Endokarditis oder degenerativ bedingte Kalzifizierungen

- Myxome oder Fibroelastome des Herzens oder an den Tumoren entstandene Thromben

- Parietalthromben im Bereich von myokardialen Infarktzonen aufgrund entzündlich veränderten Endokards oder ventrikulärer Akinesiezonen oder Aneurysmata

- Offenes Foramen ovale und/oder hypermobile Vorhofsepten

- Vorhof- oder Ventrikelseptumdefekte

Studien bei über 7000 Patienten eine äquieffektive Verhinderung thrombembolischer Ereignisse bei geringerer Blutungsrate nachgewiesen werden. Derzeit beurteilen die Zulassungsbehörden die Substanz für die Langzeittherapie, wobei asymptomatische Leberwerterhöhungen bei einigen Patienten Anlass zur Sorge geben.

Aufgrund der schwierigen Antikoagulation mit Vitamin-K-Antagonisten und der erhöhten Gefahr schwerer Blutungskomplikationen werden nur ungefähr die Hälfte der Patienten mit einer prinzipiellen Indikation für eine Antikoagulation wirklich mit oralen Antikoagulantien behandelt. Dies gilt vor allem für ältere Patienten, bei denen neben Complianceproblemen bei der Medi-

\section{Abb. 3 Persistierendes Foramen ovale}

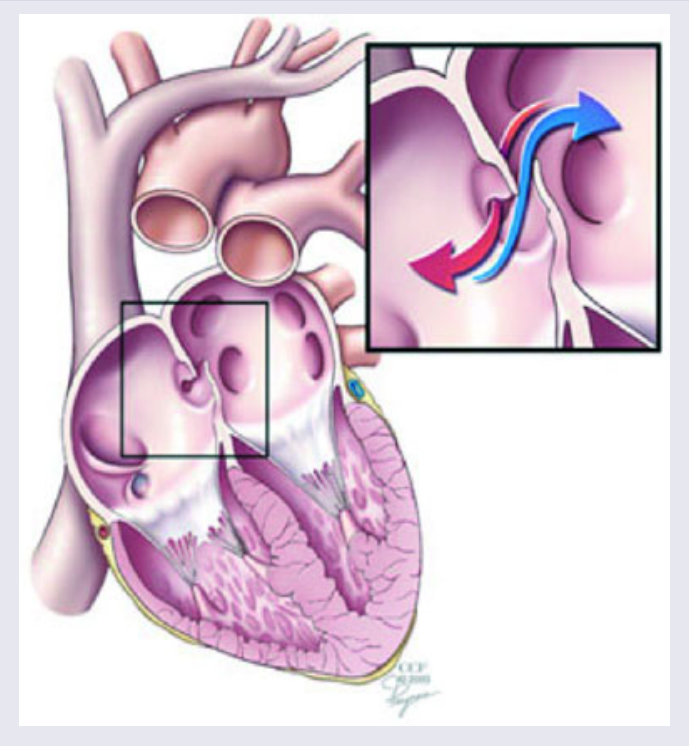

kamenteneinnahme und regelmäßigen INR-Kontrollen auch erhöhte Blutungsrisiken aufgrund von Gangunsicherheit und Stürzen befürchtet werden. Bei der Indikationsstellung zur oralen Antikoagulationsbehandlung sollte dabei vor allem das wahrscheinliche Schlaganfallrisiko in Betracht gezogen und gegen das individuelle Blutungsrisiko abgewogen werden. Bei der Berechnung des Schlaganfallrisikos helfen die Daten der Framingham-Studie, die zum sogenannten Framingham-Risk-Score geführt haben (Abb. 2).

\section{Das symptomatische Foramen ovale Epidemiologie}

Ein offenes Foramen ovale (PFO) ist mit einer Prävalenz um 30\% ein relativ häufiger Befund bei einer gesunden Normalpopulation wie in Autopsiestudien nachgewiesen werden konnte. Bei jüngeren Patienten $(<55 \mathrm{~J})$ mit einem Hirninfarkt unklarer Ursache liegt die Inzidenz bei Vorliegen eines PFO bei bis zu $50 \%$. Das Risiko, bei Vorliegen eines offenen Foramen einen Schlaganfall zu bekommen, scheint von der Größe des Defekts abzuhängen (4) und ist bei zusätzlichem Vorliegen eines Vorhofseptumaneurysmas (VSA) noch einmal deutlich erhöht. In einer Metaanalyse (10) aus Fall-Kontroll-Studien ergab sich für das Auftreten eines Schlaganfalls bei Vorliegen eines PFO eine Odds Ratio (OR) von 1,83, für die Kombination aus PFO und VSA eine OR von 5,2. Langzeituntersuchungen zur Identifikation des primären Risikos, insbesondere bei Vorliegen eines isolierten PFOs, fehlen derzeit, dennoch berechnen Kraywinkel et al. (6), dass jeder dritte Schlaganfall in der Altersgruppe der unter 55-Jährigen auf ein PFO zurückzuführen sein dürfte.

\section{Entstehung}

Das persistierende Foramen ovale ist eine kulissenartige Öffnung die zwischen dem linksatrialen Septum primum und dem rechtsatrialen Septum secundum im Bereich der Fossa ovalis nach unvollständiger Fusion der beiden Septen besteht. Im fetalen Kreislauf liegt hier ein physiologischer Blutfluss vom rechten zum linken Vorhof mit oxygeniertem Blut aus der Plazenta vor. Mit Unterbrechung des plazentaren Kreislaufes bei der Geburt kommt es über eine linksatriale Druckerhöhung in aller Regel zu einem raschen Verschluss dieser Öffnung. Septum primum und secundum verwachsen miteinander. Geschieht dies unvollständig, liegt ein persistierendes Foramen ovale (PFO) vor (Abb. 3).

\section{Pathomechanismus}

Der vorwiegend favorisierte kausale Zusammenhang zwischen einem PFO und dem Auftreten eines thrombembolischen Schlaganfalls ist die paradoxe Embolie. Am häufigsten scheint eine vor dem Ereignis aufgetretene tiefe Beinvenenthrombose zu einer Lungenembolie mit konsekutiver pulmonalarterieller Druckerhöhung zu führen; in der Folge kommt es bei Vorliegen eines PFO zumindest passager zu einem Rechts-Links-Shunt, der einen Transfer von Thromben durch das Vorhofseptum und im weiteren eine periphere Embolisation begünstigt. Tatsächlich finden sich bei über $20 \%$ dieser Patienten Thromben im BeinBeckenbereich. Desweiteren erscheint eine erhöhte lokale Thrombogenität bei Vorliegen eines PFO insbesondere in Assoziation mit einem VSA möglich: in seltenen Fällen konnte ein so entstandener Thrombus bei der Passage im Sinne einer paradoxen Embolie durch das Vorhofseptum dargestellt werden (5). Ein weiterer postulierter Pathomechanismus steht im Zusammenhang mit der Tatsache, dass insbesondere bei Vorliegen der Kombination von PFO und VSA eine erhöhte Inzidenz von Vorhofarrhythmien besteht, die beispielsweise durch eine erhöhtes atriales „streching“ bei einem Valsalva-Manöver induziert werden können (2).

\section{Diagnosestellung}

Die Methode der Wahl zur Identifikation eines PFO ist die Durchführung einer transösophagealen Echokardiografie (8), wobei gelegentlich auch die Darstellung durch eine transthorakale Untersuchung gelingt. Zur Identifikation eines Rechts-Links-Shunts über das PFO 
wird typischerweise Echokontrastmedium über eine periphere Vene injiziert. Der Patient führt simultan dazu ein Vasalva-Manöver durch. Bei Vorliegen eines PFO kommt es zu einem Übertritt von „Bläschen“ in den linken Vorhof. Dabei kann die Quantität des Übertritts als Maß für die Größe des PFOs gelten. In aktuellen Publikationen wird zunehmend die Rolle der transkraniellen Doppleruntersuchung (ebenfalls mit Injektion von Kontrastmedium unter Valsalva-Manöver) in Diagnostik und Verlaufskontrolle bei PFO hervorgehoben.

\section{Therapie}

Evidenzbasierte Strategien zur Sekundärprophylaxe bei Patienten mit Schlaganfall und einem PFO gibt es nicht. Dennoch besteht heute bei Vorliegen eines VSA in Kombination mit einem PFO bei nachgewiesener oder vermuteter paradoxer Embolie eine Indikation zum PFO-Verschluss, wobei der kathetherinterventionelle Verschluss mit einem Schirmchen die Operation am offenen Herzen mit dem Nahtverschluss des Defekts abgelöst hat (9). Die perkutan interventionelle Platzierung eines Schirmchens ist heute ein relativ kurz dauernder Eingriff mit einer periinterventionellen Komplikationsrate von um $1 \%$. Das weltweit am häufigsten verwendete System ist der selbstzentrierende AMPLATZERDoppelschirm (Abb. 4), der aus Nitinoldraht und einer Dacron-Gewebeeinlage besteht, es kommen jedoch auch andere Systeme zur Anwendung, wobei der primäre Verschluss praktisch immer gelingt.

Bei den follow-up-Untersuchungen nach Okkluderplatzierung werden noch häufiger geringe Restshunts nachgewiesen, wobei diese im Zeitverlauf abnehmen. Die Wahrscheinlichkeit eines erneuten thrombembolischen Ereignisses wird nach Verschluss zwischen 1,7 und $4 \%$ pro Jahr angegeben (7). Nach den Empfehlungen der Deutschen Gesellschaft für Neurologie (3) ist auch bei Schlaganfallrezidiv unter Phenprocoumon und bei Vorliegen einer Kontraindikation zur Antikoagulation ein Schirmchenverschluss zu empfehlen. Patienten mit einem isolierten PFO sollten zur Rezidivprophylaxe Acetylsalicylsäure erhalten, bei einem darunter auftretenden Rezidiv sollte eine Behandlung mit Phenprocoumon erfolgen. Derzeit laufen drei multizentrische, randomisierte Studien, die Ergebnisse zum Vergleich von medikamentöser und interventioneller Therapie bei diesen Patienten liefern sollen (RESPECT/USA; Closure/USA und PCTrial/Europa). Die Verbesserung und Fortentwicklung der Verschlusssysteme sowie der Einsatz neuer Medikamente (z.B. Thrombinantagonisten) werden Einfluss auf zukünftige Therapiestrategien haben.

\section{Cardiac Causes of Stroke: Atrial Fibrillation and Patent Foramen Ovale}

Atrial fibrillation and patent foramen ovale are the most common causes of cardioembolic strokes. Clinical predictors of a high systemic thromboembolic risk and thus of developing a stroke, are advancing age, a prior stroke, diabetes mellitus, arterial hypertension, previous myocardial infarction, and cardiac failure. As preventive measures, permanent dose-adapted anticoagulation has proved to be effective, whereas the administration of acetylsalicylic acid alone has proved only mildly effective. The combination of clopidogrel and acetylsalicylic acid, as well as new medications, for example, factor Xa antagonists, are currently being investigated. In stroke patients with a patent foramen ovale, or in whom anticoagulation is contraindicated, closure of the foramen is now considered to be required in those who have already had an embolus.

\section{Key Words}

stroke - atrial fibrillation - patent foramen ovale - prevention - treatment whereas

\section{Literatur}

1. Barnett $H$, Eliasziw $M$, Meldrum $H$. Drugs and surgery in the prevention of ischemic stroke. N Engl J Med 1995; 332: 238-248

2. Berthet K, Lavergne T, Cohen A. Significant association of atrial vulnerability with atrial septal abnormalities in young patients with ischemic stroke of unknown cause. Stroke 2000; 31: 398-4

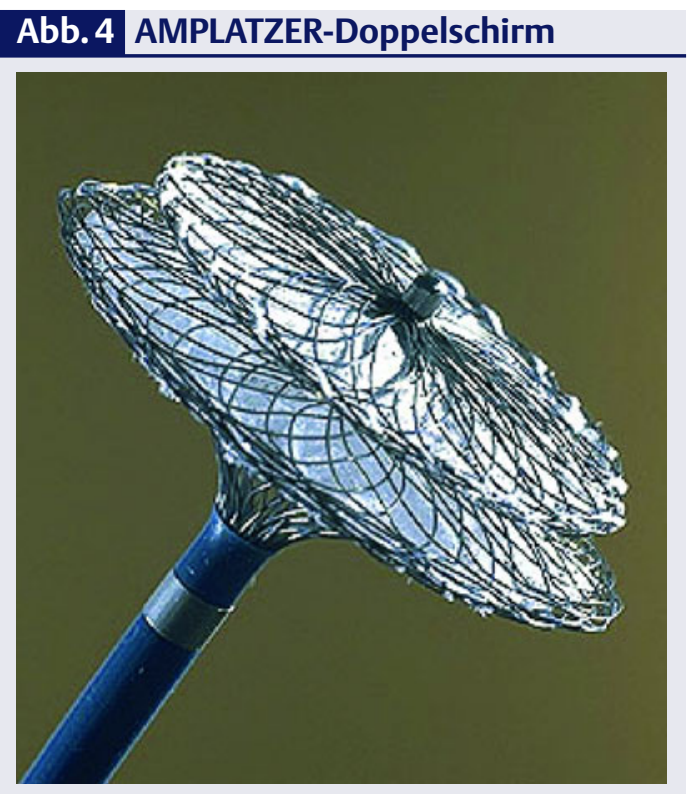

3. Deutsche Gesellschaft für Neurologie 2005. Leitlinie Primäre und sekundäre Prävention der zerebralen Ischämie. www.dgn.de

4. Homma S, Sacco RL, Di Tullio MR. Effect of medical treatment in stroke patients with patent foramen ovale: patent foramen ovale Cryptogenic Stroke Study. Circulation 105: 2625-2631

5. Koullias G, Elefteriades JA et al. Massive Paradoxical Embolism caught in the Act. Circulation 2004; 109: 3056-3057

6. Kraywinkel K, Jauss $M$, Diener $\mathrm{HC}$. Offenes Foramen ovale, Vorhofseptumaneurysma und Hirninfarkt - Übersicht über die bisherige Evidenzlage. Nervenarzt. Onlineausgabe 2. Feburar 2005

7. Krumsdorf U, Ostermayer S, Billinger $\mathrm{K}$ Incidence an clinical course of thrombus formation formation on atrial septal defect and patent foramen ovale closure devices in 1000 consecutive patients ] Am Coll Cardiol 2004; 43: 302-309

8. Mas JL, Arquizan C, Lamy C. Recurrent cerebrovascular events associated with patent foramen ovale, atrial septal aneurysm, or both. N Engl J Med 2001; 345: 1740-1746 9. Meier B. Closure of patent foramen ovale: technique, pitfalls, complications and follow up. Heart 2005; 91: 444-448

10. Overell JR, Bone I, Lees KR. Interatria septal abnormalities and stroke: a metaanalysis of case-control studies. Neurology 2000; 55: 1172-1179

11. Wang TJ, Massaro JM, Levy D et al. A risk score for predicting stroke or death in individuals with new-onset atrial fibrillation in the community: the Framingham heart study. JAMA 2003 (27. August); 290: 1049-56

\section{Korrespondenzadresse:}

Prof. Dr. Harald Darius

Vivantes Klinikum Neukölln

Klinik für Kardiologie und konservative Intensivmedizin

Rudower Straße 48, 12351 Berlin 